\title{
Semiparametric Indirect Utility and Consumer Demand
}

\author{
Krishna Pendakur ${ }^{\mathrm{a}}$, Michael Scholz ${ }^{\mathrm{b}}$, Stefan Sperlich*,b,1 \\ ${ }^{a}$ Department of Economics, Simon Fraser University, Burnaby, BC, Canada, V5A $1 S 6$ \\ ${ }^{b}$ Institut für Statistik und Ökonometrie, Georg-August Universität Göttingen, Platz der \\ Göttinger Sieben 5, 37073 Göttingen, Germany
}

\begin{abstract}
We consider a semiparametric model of consumer demand. In our model, the indirect utility function is specified as a partially linear model, where utility is nonparametric in expenditure and parametric (with fixed- or varying-coefficients) in prices. Because we start with a model of indirect utility, rationality restrictions like homogeneity and Slutsky symmetry are easily imposed. The resulting model for expenditure shares (as function of expenditures and prices) is locally given by a fraction whose numerator is partially linear, but whose denominator is nonconstant and given by the derivative of the numerator. The basic insight is that given a local polynomial model for the numerator, the denominator is given by a lowerorder local polynomial. The model can thus be estimated using modified versions of local polynomial modeling techniques. Monte Carlo evidence that the proposed techniques work is provided as well as an implementation of the model on Canadian consumer expenditure and price micro-data.
\end{abstract}

\footnotetext{
${ }^{*}$ Corresponding author: S. Sperlich, Institut für Statistik und Ökonometrie, Georg-August Universität Göttingen, Platz der Göttinger Sieben 5, 37073 Göttingen, Germany. Phone: +49551397350, Fax: +49551397279

Email addresses: stefan.sperlich@wiwi.uni-goettingen.de (Stefan Sperlich), stefan.sperlich@wiwi.uni-goettingen.de (Stefan Sperlich)

${ }^{1}$ The first author acknowledges the finanical support of the Social Sciences and Humanities Research Council of Canada through its Standard Research Grants program. The last author acknowledges support by the DFG project FOR916.
} 


\section{Introduction}

The specification and estimation of consumer demand systems, defined as the relationship between demands, prices and expenditures, presents many long-standing problems in econometric theory. Recent work has focused on the inclusion of highly nonlinear relationships between demand and total expenditure into empirical models of consumer demand. Since typical consumer demand microdata have a large amount of variation in total expenditure across consumers, complex relationships between demand and expenditure might potentially be identified. Unfortunately, because consumer demand models must satisfy a set of nonlinear cross-equation rationality restrictions (see [4] and [22] for original references) also known as the Slutsky symmetry restrictions, such complex relationships have been hard to incorporate. In this paper, we present a semiparametric approach to the consumer demand problem which allows for the simple imposition of the Slutsky symmetry restrictions. We use a flexible nonparametric estimation method in the total expenditure direction - where the data provide a lot of information - to get arbitrarily flexible Engel curves. However, in the price directions - where the data are less rich - we propose a parametric structure.

Nonparametric approaches to consumer demand started by considering the Engel curve, defined as the relationship of expenditure-shares commanded by each good to the total expenditure of the consumer, at fixed vectors of prices. That is, they considered only 1 nonparametric direction and held the others fixed. Work by [3] and [2] revealed considerable complexity in the shapes of Engel curves. A fully nonparametric approach which consider both price and expenditure directions together and which allows for the imposition of rationality restrictions, has recently been developed by [6]. Here, the shape of the demand curves is not restricted, but curse of dimensionality rears its head: in a world with $M$ price directions and 1 expenditure direction, the researcher faces an $M+1$ dimensional problem. Even if homogeneity, another rationality condition, is imposed, the researcher still faces an $M$ dimensional problem, which is still very high in typical applications.

On the other hand, parametric approaches like the popular Almost Ideal [4], Translog [10] and Quadratic Almost Ideal [1] demand models typically impose strict limits on the functional complexity of Engel curves. In these cases, Engel curves must be linear, nearly linear, or quadratic, respectively, in the log of total expenditure. This lack of complexity is driven by the need for these parametric models to satisfy the Slutsky symmetry restrictions.

A major use of consumer demand systems is in policy analysis: demand systems are used to assess whether or not indirect tax changes are desirable, and are used to assess changes in the cost-of-living. In this regard, lack of complexity has costs: in particular, if the Engel curve is wrong, then all consumer surplus calculations (including cost-of-living calculations) are also wrong. For example, [1] and [12] show that the false imposition of linear and quadratic Engel curves, respectively, can lead to very misleading estimates of behavioural and welfare responses to indirect tax changes.

In between the fully nonparametric and the fully parametric approaches, we have the realm of semiparametric econometrics. Two recent papers have explored this area, accommodating the need for the structural model to satisfy Slutsky symmetry. [12] propose a fully parametric approach which satisfies rationality restrictions and for which Engel curves can be arbitrarily complex. Because their model allows for arbitrarily complex, i.e. nonparametric, Engel curves but parametrically restricted dependence of expenditure shares on prices, it may be interpreted as semiparametric. However, their approach relies critically on a particular interpretation of the 
'error term' in the regression: it must represent unobserved preference heterogeneity, and thus cannot be measurement error or any other deviations from optimal choice on the consumer's part. [15] propose a semiparametric model which allows for these latter interpretations of the role of the error term, does not restrict the shape of Engel curves, and incorporates price effects either parametrically or semiparametrically (via varying coefficients, cf. [19]). While potentially appealing, implementation of their model requires numerical inversions of unknown functions. Both of these semiparametric approaches address the curse of dimensionality: they each have just 1 nonparametric dimension rather than $M+1$ nonparametric dimensions.

The present paper is a semiparametric contribution, similar in spirit to [15], but does not require the researcher to undertake numerical inversions of unknown functions. [15] write down a model in which expenditure-shares are nonparametric in utility - an unobserved regressor - and parametric in log-prices. The familiarity of this partially linear form makes the model appealing, but the unobserved regressor (utility) must be constructed under the model via numerical inversion of the (unknown) cost function. In the present paper, we write down a model in which utility is nonparametric in log-expenditure and parametric in log-prices. This results in a model of expenditure-shares with only 1 nonparametic dimension which is locally nonlinear but has no unobserved or generated regressors.

The local nonlinearity of our model of expenditure-shares is driven by the fact that we start by modeling indirect utility as partially linear, and since Roy's Identity gives expenditure shares as the ratio of derivatives of indirect utility, expenditure shares in our model are given by a ratio. This ratio has model parameters in both the numerator and denominator. In particular, the ratio which characterises expenditure shares has nonparametric functions in the numerator and their derivatives in the denominator. Because local estimators have to be evaluated locally at a large number of points, a locally nonlinear model, which may take a long time to evaluate at each point, is typically not very useful. Our basic insight is if one models the numerator as a local polynomial, the denominator - which is comprised of derivatives of the numerator - is just a lower-order local polynomial. This fact suggests a natural iterative procedure to estimate the model. Our algorithm is computationally efficient and numerically robust. Furthermore, large data sets can be handled in acceptable time, and the results are readily interpreted.

For the nonparametric part of the model we use an univariate local linear smoother on transformed data, a method that can be easily applied in empirical research. To accommodate the parametric part of the model, we use a restricted (to meet the Slutsky symmetry) least squares estimator. Profiled methods (see e.g. [20] or [18]) are applicable here, but not neccessary in practise due to the extremely low correlation between the covariates, i.e. between relative prices and total expenditures. In the applied part of the paper we show the power of our method in a short simulation study, and implement the model with Canadian price and expenditure data.

The paper is organised as follows. In section 2 we introduce the model specification. In section 3 we discuss the basic estimation idea and give the associated algorithm. The empirical part of the paper can be found in section 4, where we present the estimation results of the Canadian microdata and the simulation study. Here, we find that some expenditure-share equations show quite a lot of nonlinearity, i.e. these are S-shaped or even more complex. Section 5 concludes the present work and discusses some possible extensions. 


\section{A Semiparametric Model for Indirect Utility}

Define the indirect utility function $V(p, x)$ to give the maximum utility attained by a consumer when faced with a vector of $\log$-prices $p=\left(p^{1}, \ldots, p^{M}\right)$ and $\log$ total expenditure $x$. Let the expenditure share of a good be defined as the expenditure on that good divided by the total expenditure available to the consumer. Denote $w=\left(w^{1}, \ldots, w^{M-1}\right)$ as the vector of expenditure share functions and note that since expenditure shares sum to $1, w^{M}=1-\sum_{j=1}^{M-1} w^{j}$. Let $\left\{W_{i}^{1}, \ldots, W_{i}^{M}, P_{i}^{1}, \ldots, P_{i}^{M}, X_{i}\right\}_{i=1}^{N}$ be a random vector giving the expenditure shares, $\log$-prices and log-total expenditure of a population of $N$ individuals.

\subsection{A Partial Linear and Varying Coefficient Model for Indirect Utility}

We consider two semiparametric specifications of the indirect utility function. First, we consider a partially linear (or, fixed-coefficient) specification of the form

$$
V(p, x)=x-\sum_{k=1}^{M} f^{k}(x) p^{k}-\frac{1}{2} \sum_{k=1}^{M} \sum_{l=1}^{M} a^{k l} p^{k} p^{l}
$$

or, in matrix notation,

$$
V(\mathbf{p}, x)=x-\mathbf{f}(x)^{\prime} \mathbf{p}-\frac{1}{2} \mathbf{p}^{\prime} \mathbf{A} \mathbf{p}
$$

where $\mathbf{f}=\left(f^{1}, \ldots, f^{M}\right)^{\prime}$ are unknown differentiable functions of log-total expenditure and $\mathbf{A}=\left\{a^{k l}\right\}_{k, l=1}^{M}$ are symmetric parameters satisfying $a^{k l}=a^{l k}$. Second, we consider the varying-coefficient extension of this model:

$$
V(p, x)=x-\sum_{k=1}^{M} f^{k}(x) p^{k}-\frac{1}{2} \sum_{k=1}^{M} \sum_{l=1}^{M} a^{k l}(x) p^{k} p^{l},
$$

or, in matrix notation,

$$
V(\mathbf{p}, x)=x-\mathbf{f}(x)^{\prime} \mathbf{p}-\frac{1}{2} \mathbf{p}^{\prime} \mathbf{A}(x) \mathbf{p} .
$$

The motivation for these models is as follows. In real-world applications, there is typically a large amount of observed variation in total expenditures, so one may reasonably hope to identify a nonparametric component in that direction. However, typical micro-data sources do not have nearly as much variation in the price direction, which suggests that partially linear modelling might describe these effects sufficiently well. If additionally, the researcher feels that more may be identified on the strength of observed variation, the varying-coefficients model allows price effects in the model (3) to be different at different expenditure levels. This would seem to be a pure advantage of the varying coefficients approach. However, in practise, this extension seriously increases the variance and computational cost of the estimates. In particular, the algorithm for model (3) is about five times slower than the one for model (1). The important feature here is that nonparametric dimensionality is kept to 1 in both models.

\subsubsection{Rationality Restrictions: Homogeneity}

Rationality is comprised of three conditions: homogeneity, symmetry and concavity. As is common in this literature, we will deal only with homogeneity and symmetry. 
First consider homogeneity, which is sometimes referred to as 'no money illusion'. If consumers do not suffer from money illusion, then scaling prices and expenditures by the same factor cannot affect utility. This requires that indirect utility is homogeneous of degree zero in (unlogged) prices and expenditure, which implies $V(p, x)=V(p+\lambda, x+\lambda)$ for all $\lambda$. This can be achieved by dividing all prices and expenditure by the price of the $M$-th expenditure category. Note that we use logarithms, so we subtract $p^{M}$ :

$V(p, x)=\left(x-p^{M}\right)-\sum_{k=1}^{M-1} f^{k}\left(x-p^{M}\right) \cdot\left(p^{k}-p^{M}\right)-\frac{1}{2} \sum_{k=1}^{M-1} \sum_{l=1}^{M-1} a^{k l}\left(p^{k}-p^{M}\right)\left(p^{l}-p^{M}\right)$

in model (1) and analogously in model (3). The sums go only to $M-1$ because the $M$-th element of the sums is zero. Using $\tilde{x}=x-p^{M}, \tilde{p}^{j}=p^{j}-p^{M}$ and $\tilde{p}=\left(\tilde{p}^{1}, \ldots, \tilde{p}^{M-1}\right)$ we may write

$$
V(\tilde{p}, \tilde{x})=\tilde{x}-\sum_{k=1}^{M-1} f^{k}(\tilde{x}) \cdot \tilde{p}^{k}-\frac{1}{2} \sum_{k=1}^{M-1} \sum_{l=1}^{M-1} a^{k l} \tilde{p}^{k} \tilde{p}^{l}
$$

Given an indirect utility function, Marshallian (uncompensated) expenditure share equations may be recovered via the logarithmic version of Roy's Identity: $w^{j}(p, x)=$ $\left[\partial V(p, x) / \partial p^{j}\right] /[\partial V(p, x) / \partial V x]$. In the following we will only think in vectors of length $(M-1)$, because the last budget share is defined by the 'adding up' condition:

$$
w^{M}(\tilde{p}, \tilde{x})=1-\sum_{i=1}^{M-1} w^{i}(\tilde{p}, \tilde{x})
$$

guaranteeing that $\sum_{j=1}^{M} w^{j}=1$ for all $(\tilde{p}, \tilde{x})$.

Applying Roy's identity to our indirect utility function, we get the uncompensated expenditure share equations

$$
\mathbf{w}(\tilde{\mathbf{p}}, \tilde{x})=\frac{\mathbf{f}(\tilde{x})+\mathbf{A} \tilde{\mathbf{p}}}{1-\nabla_{\tilde{x}} \mathbf{f}(\tilde{x})^{\prime} \tilde{\mathbf{p}}},
$$

for the fixed-coefficients model (1), and

$$
\mathbf{w}(\tilde{\mathbf{p}}, \tilde{x})=\frac{\mathbf{f}(\tilde{x})+\mathbf{A}(\tilde{x}) \tilde{\mathbf{p}}}{1-\nabla_{\tilde{x}} \mathbf{f}(\tilde{x})^{\prime} \tilde{\mathbf{p}}-\frac{1}{2} \tilde{\mathbf{p}}^{\prime} \nabla_{\tilde{x}} \mathbf{A}(\tilde{x}) \tilde{\mathbf{p}}},
$$

for the varying-coefficients model (3). Here, $\nabla_{\tilde{x}} \mathbf{f}(\tilde{x})$ is the $(M-1)$ vector of the derivatives of $\mathbf{f}(\tilde{x})$, and $\nabla_{\tilde{x}} \mathbf{A}(\tilde{x})$ is the $(M-1) \times(M-1)$ matrix function equal to the derivatives of $\mathbf{A}$.

These expressions for budget shares have a nice feature in comparison to [15]. Whereas their model for expenditure shares uses a nonparametric function of a generated regressor, the expression above uses only observed regressors. However, in comparison to [15], which is a partially linear model, the above expression is partially linear only in the numerator. The presence of the denominator makes it seem difficult to implement. However, as we show below, use of local polynomial estimators makes this problem manageable.

\subsubsection{Rationality Restrictions: Slutsky Symmetry}

The imposition of Slutsky symmetry requires that $a^{l k}=a^{k l}\left(a^{l k}(x)=a^{k l}(x)\right)$ for all $k, l$, or equivalently, that $\mathbf{A}=\mathbf{A}^{\prime}\left(\mathbf{A}(x)=\mathbf{A}(x)^{\prime}\right)$. To see this, start with the 
definition of the Slutsky matrix, whose elements are given by

$$
s_{i j}=\frac{\partial h^{i}}{\partial b^{j}}=\frac{\partial g^{i}}{\partial y} \cdot q^{j}+\frac{\partial g^{i}}{\partial b^{j}},
$$

where $h^{i}$ denotes the Hicksian demand, $g^{i}$ the Marshallian demand, $y$ total expenditure, $b^{j}$ the price, and $q^{j}$ the quantity of good/category $j$. With $q^{i}=g^{i}=w^{i} \cdot y / b^{i}$, where $g^{i}$ and $w^{i}$ are functions of $y, b^{1}, \ldots, b^{M}$, we get

$$
\frac{\partial g^{i}}{\partial y}=\frac{\partial w^{i}}{\partial y} \cdot \frac{y}{b^{i}}+\frac{w^{i}}{b^{i}} \quad \text { and } \quad \frac{\partial g^{i}}{\partial b^{j}}=\frac{\partial w^{i}}{\partial b^{j}} \cdot \frac{y}{b^{i}} .
$$

Using the equations in (8) and (9) we can write the difference of $s_{i j}-s_{j i}$, for $i, j=1, \ldots, M-1$, as

$$
s_{i j}-s_{j i}=\left(\frac{\partial w^{i}}{\partial y} \cdot w^{j}-\frac{\partial w^{j}}{\partial y} \cdot w^{i}\right) \frac{y^{2}}{b^{i} b^{j}}+\left(\frac{\partial w^{i}}{\partial b^{j}} \cdot \frac{y}{b^{i}}-\frac{\partial w^{j}}{\partial b^{i}} \cdot \frac{y}{b^{j}}\right) .
$$

With the abbreviations $T^{i}=\sum a^{i k} \tilde{p}^{k}, S=1-\sum \partial f^{k} / \partial \tilde{x} \cdot \tilde{p}^{k}$ and $f^{i}=f^{i}(\tilde{x})$, we can rewrite (6) in the following way

$$
w^{i}=\frac{f^{i}+T^{i}}{S},
$$

but note that $w^{i}$ depends on $\tilde{x}=\log y-\log b^{M}, \log$-total expenditure, and $\tilde{p}^{j}=$ $\log b^{j}-\log b^{M}$ the $\log$-prices for $j=1, \ldots, M-1$. Now we can differentiate (11) w.r.t. total expenditure and the $j$-th price, and obtain with $U=\sum \partial^{2} f^{k} / \partial \tilde{x}^{2} \cdot \tilde{p}^{k}$

$$
\frac{\partial w^{i}}{\partial y}=\frac{\frac{\partial f^{i}}{\partial \tilde{x}} \cdot S+\left(f^{i}+T^{i}\right) \cdot U}{y S^{2}} \quad \text { and } \quad \frac{\partial w^{i}}{\partial b^{j}}=\frac{a^{i j} S+\left(f^{i}+T^{i}\right) \cdot \frac{\partial f^{j}}{\partial \tilde{x}}}{b^{j} S^{2}} .
$$

Plugging-in these results and equation (11) in (10), we get immediately that $s_{i j}-$ $s_{j i}=0$ if $a_{i j}=a_{j i}$.

\section{Estimation of the Models}

The main advantage of the above models is the enormous dimension reduction for the nonparametric part of the estimation procedure. Instead of $M+1$ or $M$ dimensions for a fully nonparametric model, we face only functions which are one dimensional in total-expenditure. Thus, we circumvent the curse of dimensionality.

In the following sections, we show how to estimate the $M-1$ vector $\mathbf{w}(\tilde{\mathbf{p}}, \tilde{x})$ under the model. Such estimates satisfy "adding-up" by construction, since $w^{M}(\tilde{p}, \tilde{x})=$ $1-\sum_{i=1}^{M-1} w^{i}(\tilde{p}, \tilde{x})$. They satisfy homogeneity ("no money illusion") by construction, due to the use of normalised prices and expenditures as regressors. Finally, they satisfy Slutsky symmetry by construction, because the matrix $\mathbf{A}$ (or $\mathbf{A}(\tilde{x})$ ) can easily be restricted to be a symmetric matrix.

A more difficult problem is to restrict the estimated budget shares to be everywhere in the range $[0,1]$. This problem is referred to as the "global regularity" problem in the literature on consumer demand. Roughly speaking, demand systems that are not homothetic - that is, those which have budget shares which respond to total expenditure - cannot typically be globally regular without restricting either the domain of $p, x$ or the domain of model error terms in ad hoc ways. See [17] for a discussion of the former, and [12] for discussion of the latter. We will judge our 
estimates in terms of "local regularity", that is, in terms of whether or not estimated budget shares are in the range $[0,1]$ in a $p, x$ domain of interest. In particular, when $\mathbf{p}=\mathbf{0}_{M}$, we have in both the fixed-coefficients and varying-coefficients model that

$$
\mathbf{w}(\mathbf{p}, x)=\mathbf{w}(\tilde{\mathbf{p}}, \tilde{x})=\mathbf{f}(\tilde{x})=\mathbf{f}(x) .
$$

Thus, the estimated functions $\mathbf{f}(x)$ characterise budget shares over a domain spanned by $x$ with log-prices fixed at $\mathbf{0}_{M}$. If these estimated functions lie within $[0,1]$, then we say that our estimates are "locally regular" in this sense. Note also that the vast majority of the parametric literature on estimating expenditure systems does not tackle this problem due to its complexity in practice. In the context of nonand semiparametric modeling, we are only aware of [13] who estimate censored expenditures in a single equation context.

\subsection{Basic Ideas}

The basic idea of estimating the unknown nonparametric functions $f^{j}$ and the (potentially varying) coefficients $a^{j k}, j, k=1, \ldots, M-1$, consists of iteratively solving minimization problems, where the iteration is necessary only for the nonparametric part of the model. We use local linear kernel estimators for the nonparametric part, and, in case of the fixed-coefficients model (1), least squares for the parametric coefficients. Obtaining estimates consistent with Slutsky symmetry is via the use of (linearly) restricted least squares for the parametric part.

Keeping the dependence on $\tilde{x}$, we may approximate

$$
\begin{aligned}
\mathbf{f}(t) & \approx \mathbf{f}(\tilde{x})+\nabla_{\tilde{x}} \mathbf{f}(\tilde{x})(t-\tilde{x}) \\
& \approx \alpha(\tilde{x})+\beta(\tilde{x})(t-\tilde{x}),
\end{aligned}
$$

where $\alpha(\tilde{x})$ and $\beta(\tilde{x})$ are the local level and derivative of $\mathbf{f}(t)$. Then, for the partial linear model the local problem is

$$
\begin{gathered}
\min _{\alpha(\tilde{x}), \beta(\tilde{x}), \mathbf{A}} \sum_{i=1}^{N} \mathbf{e}_{i}^{\prime} \mathbf{\Omega} \mathbf{e}_{i}, \\
\mathbf{e}_{i} \equiv \mathbf{w}_{i}-\frac{\alpha(\tilde{x})+\left(\tilde{x}_{i}-\tilde{x}\right) \beta(\tilde{x})+\mathbf{A} \tilde{\mathbf{p}}_{i}}{1-\beta(\tilde{x})^{\prime} \tilde{\mathbf{p}}_{i}},
\end{gathered}
$$

where $\boldsymbol{\Omega}$ is an $(M-1) \times(M-1)$ weighting matrix.

Similarly, for the varying coefficient model (7), the local problem in the neighbourhood of each given $\tilde{x}$ is

$$
\begin{gathered}
\min _{\alpha(\tilde{x}), \beta(\tilde{x}), \boldsymbol{\Gamma}(\tilde{x}), \Delta(\tilde{x})} \sum_{i=1}^{N} \mathbf{e}_{i}^{\prime} \boldsymbol{\Omega} \mathbf{e}_{i}, \\
\mathbf{e}_{i} \equiv \mathbf{w}_{i}-\frac{\alpha(\tilde{x})+\left(\tilde{x}_{i}-\tilde{x}\right) \beta(\tilde{x})+\boldsymbol{\Gamma}(\tilde{x}) \tilde{\mathbf{p}}_{i}+\left(\tilde{x}_{i}-\tilde{x}\right) \boldsymbol{\Delta}(\tilde{x}) \tilde{\mathbf{p}}_{i}}{1-\beta(\tilde{x})^{\prime} \tilde{\mathbf{p}}_{i}-\frac{1}{2} \tilde{\mathbf{p}}_{i}^{\prime} \boldsymbol{\Delta}(\tilde{x}) \tilde{\mathbf{p}}_{i}},
\end{gathered}
$$

where $\boldsymbol{\Omega}$ is now a different $(M-1) \times(M-1)$ weighting matrix.

Here, the imposition of homogeneity is via the use of normalised prices and expenditures (tilda'd quantities). The imposition of Slutsky symmetry is via the restriction that $\mathbf{A}=\mathbf{A}^{\prime}$, or in the varying-coefficients case, that $\mathbf{A}(x)=\mathbf{A}(x)^{\prime}$ which is achieved by restricting $\boldsymbol{\Gamma}(\tilde{x})=\boldsymbol{\Gamma}(\tilde{x})^{\prime}$ and $\boldsymbol{\Delta}(\tilde{x})=\boldsymbol{\Delta}(\tilde{x})^{\prime}$.

We could also use higher order approximations (and thus higher-order local polynomials), but for this we would need stronger assumptions on data and model (e.g. 
higher order smoothness of functions and densities). The local linear approach however nests the parametric linear model for any smoothing bandwidth without a bias. Therefore we confine ourselves to the local linear approximation. We will now be more specific but we will see that the steps are easily generalised.

\subsection{The Estimation Algorithm}

Denote $\Delta_{i}=\tilde{X}_{i}-\tilde{x}, K_{i}=K\left(\left(\tilde{X}_{i}-\tilde{x}\right) / h\right) / h$ and $d=M-1$, where $K$ is some symmetric kernel function with the usual properties and $h$ a bandwidth that controls the smoothness of the estimate.

Let us start with the minimization problem for the partial linear model (1). As above, the $\alpha^{j}$ are related to the functions $f^{j}$ at point $\tilde{x}$ and the parameters $\beta^{j}$ to its first derivatives, while the parameters $a^{j k}$ are fixed for all $\tilde{x}$ :

$$
\min _{\alpha^{j}, \beta^{j}} \sum_{j=1}^{d} \sum_{i=1}^{N}\left(W_{i}^{j}-\frac{\alpha^{j}+\Delta_{i} \beta^{j}+\sum_{k=1}^{d} a^{j k} \tilde{P}_{i}^{k}}{1-\sum_{k=1}^{d} \beta^{k} \tilde{P}_{i}^{k}}\right)^{2} K_{i} .
$$

In order to minimize, we set the first derivative equal to zero. Taking the derivative of (14) with respect to $\alpha^{j}$, and using the notations $S_{i}=1-\sum_{k=1}^{d} \beta^{k} \tilde{P}_{i}^{k}$ and $T_{i}^{j}=\sum_{k=1}^{d} a^{j k} \tilde{P}_{i}^{k}$, we solve

$$
0=\sum_{i=1}^{N}\left(W_{i}^{j}-\frac{\alpha^{j}+\Delta_{i} \beta^{j}+T_{i}^{j}}{S_{i}}\right) \frac{K_{i}}{S_{i}} .
$$

This gives immediately (for $j=1, \ldots, d$ )

$$
\alpha^{j}=\frac{\sum_{i=1}^{N} W_{i}^{j} K_{i} / S_{i}-\beta^{j} \sum_{i=1}^{N} K_{i} \Delta_{i} / S_{i}^{2}-\sum_{i=1}^{N} K_{i} T_{i}^{j} / S_{i}^{2}}{\sum_{i=1}^{N} K_{i} / S_{i}^{2}} .
$$

On the other hand, by differentiating (14) with respect to $\beta^{j}$ (again for $j=1, \ldots, d$ ), we get the equations

$$
\begin{aligned}
0= & \sum_{i=1}^{N}\left(W_{i}^{1}-\frac{\alpha^{1}+\Delta_{i} \beta^{1}+T_{i}^{1}}{S_{i}}\right) K_{i} \cdot \frac{\left(\alpha^{1}+\Delta_{i} \beta^{1}+T_{i}^{1}\right) \tilde{P}_{i}^{j}}{S_{i}^{2}}+\cdots+ \\
& \sum_{i=1}^{N}\left(W_{i}^{j}-\frac{\alpha^{j}+\Delta_{i} \beta^{j}+T_{i}^{j}}{S_{i}}\right) K_{i} \cdot \frac{\Delta_{i} S_{i}+\left(\alpha^{j}+\Delta_{i} \beta^{j}+T_{i}^{j}\right) \tilde{P}_{i}^{j}}{S_{i}^{2}}+\cdots+ \\
& \sum_{i=1}^{N}\left(W_{i}^{d}-\frac{\alpha^{d}+\Delta_{i} \beta^{d}+T_{i}^{d}}{S_{i}}\right) K_{i} \cdot \frac{\left(\alpha^{d}+\Delta_{i} \beta^{d}+T_{i}^{d}\right) \tilde{P}_{i}^{j}}{S_{i}^{2}} .
\end{aligned}
$$

This is equivalent to

$$
\begin{aligned}
0= & \sum_{k=1}^{d} \sum_{i=1}^{N}\left(W_{i}^{k}-\frac{\alpha^{k}+\Delta_{i} \beta^{k}+T_{i}^{k}}{S_{i}}\right) K_{i} \cdot \frac{\left(\alpha^{k}+\Delta_{i} \beta^{k}+T_{i}^{k}\right) \tilde{P}_{i}^{j}}{S_{i}^{2}}+ \\
& \sum_{i=1}^{N}\left(W_{i}^{j}-\frac{\alpha^{j}+\Delta_{i} \beta^{j}+T_{i}^{j}}{S_{i}}\right) K_{i} \frac{\Delta_{i}}{S_{i}} .
\end{aligned}
$$


Unfortunately, we can not solve equation (17) analytically for $\beta^{j}$. But, for our iterative purpose it is enough to consider the following implicit representation:

$$
\begin{aligned}
\beta^{j}= & {\left[\sum_{k=1}^{d} \sum_{i=1}^{N}\left(W_{i}^{k}-\frac{\alpha^{k}+\Delta_{i} \beta^{k}+T_{i}^{k}}{S_{i}}\right) K_{i} \cdot \frac{\left(\alpha^{k}+\Delta_{i} \beta^{k}+T_{i}^{k}\right) \tilde{P}_{i}^{j}}{S_{i}^{2}}+\right.} \\
& \left.\sum_{i=1}^{N}\left(W_{i}^{j}-\frac{\alpha^{j}+T_{i}^{j}}{S_{i}}\right) K_{i} \frac{\Delta_{i}}{S_{i}}\right] / \sum_{i=1}^{N} \frac{K_{i} \Delta_{i}^{2}}{S_{i}^{2}} .
\end{aligned}
$$

We use the implicit representation (18) to calculate new values for $\beta^{j}$. With them we get new $S_{i}$ so that we can find new $\alpha^{j}$ :

$$
\begin{aligned}
\beta_{\text {new }}^{j}= & {\left[\sum_{k=1}^{d} \sum_{i=1}^{N}\left(W_{i}^{k}-\frac{\alpha_{\text {old }}^{k}+\Delta_{i} \beta_{\text {old }}^{k}+T_{i, \text { old }}^{k}}{S_{i, \text { old }}}\right) K_{i} \frac{\left(\alpha_{\text {old }}^{k}+\Delta_{i} \beta_{\text {old }}^{k}+T_{i, \text { old }}^{k}\right) \tilde{P}_{i}^{j}}{S_{i, \text { old }}^{2}}\right.} \\
& \left.+\sum_{i=1}^{N}\left(W_{i}^{j}-\frac{\alpha_{\text {old }}^{j}+T_{i, \text { old }}^{j}}{S_{i, \text { old }}}\right) K_{i} \frac{\Delta_{i}}{S_{i, \text { old }}}\right] / \sum_{i=1}^{N} \frac{K_{i} \Delta_{i}^{2}}{S_{i, \text { old }}^{2}} \\
S_{i, \text { new }}= & 1-\sum_{k=1}^{d} \beta_{\text {new }}^{k} \tilde{P}_{i}^{k}, \\
\alpha_{\text {new }}^{j}= & \frac{\sum_{i=1}^{N} W_{i}^{j} K_{i} / S_{i, \text { new }}-\beta_{\text {new }}^{j} \sum_{i=1}^{N} K_{i} \Delta_{i} / S_{i, \text { new }}^{2}-\sum_{i=1}^{N} K_{i} T_{i, \text { old }}^{j} / S_{i, \text { new }}^{2}}{\sum_{i=1}^{N} K_{i} / S_{i, \text { new }}^{2}}
\end{aligned}
$$

We repeat these steps until convergence. The optimal $\mathbf{A}$ will be the one that minimizes the least squares problem. In practice, at the end of each iteration step, we solve the restricted least squares problem resulting from equation (6). With some algebra, the problem is given by

$$
W_{i}^{j} \cdot\left(1-\sum_{k=1}^{d} \beta_{i}^{k} \tilde{P}_{i}^{k}\right)-\alpha_{i}^{j}=\sum_{k=1}^{d} a^{j k} \tilde{P}_{i}^{k} .
$$

Details are given in the Appendix. Here, we see why we need $\mathbf{A}$ to be symmetric for identification: we can only identify the sum of the symmetric effects. As noted, one could think of extending this procedure using profiled estimators (calculating the $\hat{\alpha}(\cdot), \hat{\beta}(\cdot)$ for any possible $\mathbf{A}$, and afterwards estimating $\mathbf{A}$ from (21) with $\hat{\alpha_{\mathbf{A}}}(\cdot)$, $\hat{\beta_{\mathbf{A}}}(\cdot)$ ), this is computationally rather cumbersome but needless in our context due to the low dependence between prices and total expenditures.

The modification of the algorithm to take the varying coefficients $\mathbf{A}(\tilde{x})$ into account is one along ideas of [5], though it is more complex in our context. With the same local linear approximation arguments as above, we get the local problem in the neighbourhood of $\tilde{x}$ as

$$
\min _{\theta} \sum_{j=1}^{d} \sum_{i=1}^{N}\left(W_{i}^{j}-\frac{\alpha^{j}+\Delta_{i} \beta^{j}+\sum_{k=1}^{d}\left(\gamma^{j k}+\Delta_{i} \delta^{j k}\right) \tilde{P}_{i}^{k}}{1-\sum_{k=1}^{d} \beta^{k} \tilde{P}_{i}^{k}-\frac{1}{2} \sum_{k=1}^{d} \sum_{l=1}^{d} \delta^{k l} \tilde{P}_{i}^{k} \tilde{P}_{i}^{l}}\right)^{2} K_{i}
$$

with $\theta$ denoting $\alpha^{j}, \beta^{j}, \gamma^{j k}$ and $\delta^{j k}$. Note that $\gamma^{j k}$ and $\delta^{j k}$ are symmetric since we consider a symmetric matrix of functions $a^{k l}(\tilde{x})$. The minimization of $(22)$ in the usual way gives the extended algorithm in analogy to the first step of 3.2. For $\alpha^{j}$ 
and $\beta^{j}$ we proceed as before but with $S_{i}=1-\sum \beta^{k} \tilde{P}_{i}^{k}-1 / 2 \sum \sum \delta^{k l} \tilde{P}_{i}^{k} \tilde{P}_{i}^{l}$ and $T_{i}^{j}=\sum\left(\gamma^{j k}+\Delta_{i} \delta^{j k}\right) \tilde{P}_{i}^{k}$. Furthermore, we obtain

$$
\gamma^{s t}=\frac{\sum_{i=1}^{N}\left[\left(W_{i}^{s}-\frac{C_{i}^{s}}{S_{i}}\right) \tilde{P}_{i}^{t}+\left(W_{i}^{t}-\frac{C_{i}^{t}}{S_{i}}\right) \tilde{P}_{i}^{s} \mathbb{1}_{s \neq t}\right] \frac{K_{i}}{S_{i}}}{\sum_{i=1}^{N}\left[\left(\tilde{P}_{i}^{t}\right)^{2}+\left(\tilde{P}_{i}^{s}\right)^{2} \mathbb{I}_{s \neq t}\right] \frac{K_{i}}{S_{i}^{2}}}
$$

with $C_{i}^{s}=\alpha^{s}+\Delta_{i} \beta^{s}+T_{i}^{s}-\gamma^{s t} \tilde{P}_{i}^{t}$ and

$$
\begin{aligned}
& \delta^{s t}=\left[\sum_{k=1}^{d} \sum_{i=1}^{N}\left(W_{i}^{k}-\frac{\alpha^{k}+\Delta_{i} \beta^{k}+T_{i}^{k}}{S_{i}}\right) \frac{K_{i}}{S_{i}^{2}}\left(\alpha^{k}+\Delta_{i} \beta^{k}+T_{i}^{k}\right) \tilde{P}_{i}^{t} \tilde{P}_{i}^{s}+\right. \\
& \left.\sum_{i=1}^{N}\left\{\left(W_{i}^{s}-\frac{\alpha^{s}+\Delta_{i} \beta^{s}+T_{i}^{s,-t}}{S_{i}}\right) \tilde{P}_{i}^{t}+\left(W_{i}^{t}-\frac{\alpha^{t}+\Delta_{i} \beta^{t}+T_{i}^{t,-s}}{S_{i}}\right) \tilde{P}_{i}^{s}\right\} \frac{K_{i} \Delta_{i}}{S_{i}}\right] \\
& \times\left[\sum_{i=1}^{N}\left\{\left(\tilde{P}_{i}^{t}\right)^{2}+\left(\tilde{P}_{i}^{s}\right)^{2} \mathbb{I}_{s \neq t}\right\} \frac{\Delta_{i}^{2} K_{i}}{S_{i}^{2}}\right]^{-1}
\end{aligned}
$$

with $T_{i}^{s,-t}=T_{i}^{s}-\Delta_{i} \delta^{s t} \tilde{P}_{i}^{t}$.

\subsection{Practical Considerations}

One issue in an such iterative procedure is the question of adequate initial values for the nonparametric part. Here we have a convenient model feature to exploit: when we normalise prices in the sample such that $\tilde{P}_{i}=(0, \ldots, 0)$ for some group of consumers, equation (14) reduces to the well-known local linear case. That is, since $S_{i}=1$, we get the objective function

$$
\min _{\alpha^{j}, \beta^{j}} \sum_{j=1}^{d} \sum_{i=1}^{N}\left(W_{i}^{j}-\alpha^{j}+\Delta_{i} \beta^{j}\right)^{2} K_{i} .
$$

Solving this problem on the sample of consumers where $\tilde{P}_{i}=(0, \ldots, 0)$ gives us consistent estimates (though with a possibly large variance due to the small subsample size) that we can use as starting values for $\alpha^{j}$ and $\beta^{j}$. For the varying coefficient model we need additionally starting values for the $\gamma^{j k}$ and $\delta^{j k}$. As a natural choice for we use the results of the algorithm in Section 3.2 and zero for all $\delta^{j k}$ (i.e. starting in the first iteration with a simpler model but still more flexible than our partial linear one).

For the bandwidth choice, we use the same bandwidth $h$ for all expenditure categories because the functions refer to the same expenditure data in all equations. As bandwidth choice criterion one may use the usual cross-validation or plug-in rules like Silverman's (although this was constructed for density estimates rather than regression).

Recall that the $M^{\text {th }}$ equation and its Engel curve is simply a result of the homogeneity and the summing-up condition $\sum_{j=}^{M} W_{i}^{j}=1$. In practice one might therefore choose the item for which less variation in the shares is observed over the households, and in which one is less interested.

Note finally in the fixed-coefficients model (1), it is often recommended running the whole algorithm twice: first with an undersmoothing bandwidth in the nonparametric part to keep the possible smoothing bias small. The resulting estimate for the coefficient matrix $\mathbf{A}$ is kept, and used in the second run which uses a larger bandwidth for the nonparametric part to get reasonably smooth function estimates. This is of course unnecessary in the varying coefficients model (3) where we face only nonparametric functions. 


\section{Empirical Analysis}

To get an idea about the finite sample performance of the method, before we analyse household expenditures in Canada, we start with a brief simulation study. Afterwards, we also introduce a wild bootstrap procedure for further inference like the construction of confidence intervals. In particular, we introduce an original conditionally asymmetric bootstrap to guarantee that bootstrap shares are in $[0,1]$.

\subsection{A Simulation Study}

First, to generate some artificial data, we generated 33 distinct price vectors, normally distributed in each dimension, for each of 6 expenditure categories (i.e. we have 6 items with different prices in 33 regions). As in typically observed microdata, we did not allow for a wide price variety, see [11]. Summary values for these price vectors can be found in Table 1 .

Table 1: Summary of used price vectors in simulation

\begin{tabular}{lccccc}
\hline \hline & 1 & 2 & 3 & 4 & 5 \\
\hline Min & 3.905 & 3.449 & 3.763 & 0.880 & 2.794 \\
Max & 4.130 & 3.585 & 3.919 & 1.121 & 3.010 \\
Mean & 4.018 & 3.517 & 3.841 & 1.002 & 2.901 \\
Std. & 0.030 & 0.020 & 0.020 & 0.030 & 0.030 \\
\hline \hline
\end{tabular}

For 32 regions (i.e. price vectors) we uniformly draw $30 \log$-total expenditure values from the interval $[1,2]$. For the reference region (number 33) we draw 40 uniformly distributed values between one and two. In total, this gives us $N=1000$ observations. These are used to generate expenditure shares using the expenditure functions shown in Figure 1 (solid lines), price parameters given in Table 2, and normal error terms with mean zero and standard deviation 0.01 . In order to get shares which fulfill the conditions $W^{j} \in[0,1]$ and $\sum_{j} W^{j}=1$ we applied the rejection method (that is, we dropped and replaced values outside $[0,1]$ ).

Next, we estimate the functions $\alpha^{j}$ and the price parameters using our estimation algorithm introduced in Section 3.2. This is repeated 250 times (using the same functions, price parameters, and range of log-total expenditure values) to get an idea of the mean squared errors of our estimators. For the nonparametric part of the estimation procedure we used the Gaussian kernel and a data-adaptive bandwidth of $h \approx 0.034$.

In Figure 1 we have plotted the true functions (solid lines) together with intervals of $90 \%$ coverage probabilities for the estimates (dashed lines) as a result of the 250 simulation runs. On the one hand, we see pretty narrow bands which accurately capture even those functions with flat plateaus in the intermediate range (category 3 ) and with bumps (category 2). Such functions are often hard to estimate in practise. However, we also see the limits of the method: for example, boundary effects seem important. Not surprisingly, the local linear smoother can estimate perfectly, and without any bias the linear function (category 5).

In Table 3 are given the estimated parameter means, together with the standard deviations. The exactness of our simulation results in a very small total MSE of only $6.83 \cdot 10^{-6}$. 

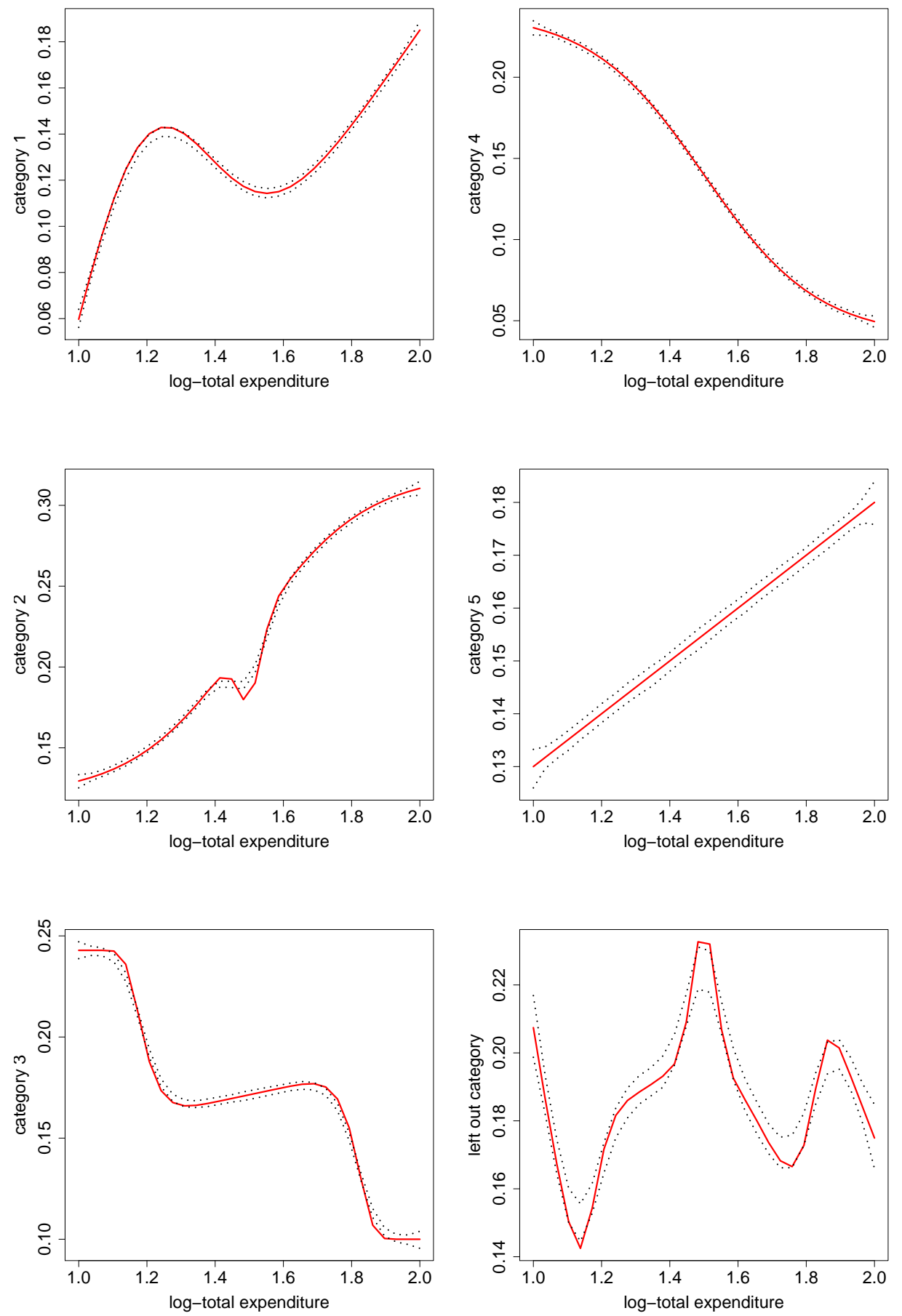

Figure 1: Simulation of 6 different budget share functions (solid line) with $90 \%$ coverage probability (dashed lines) 
Table 2: Price parameters used in the simulation

\begin{tabular}{rrrrrr}
\hline \hline & 1 & 2 & 3 & \multicolumn{1}{c}{4} & \multicolumn{1}{c}{5} \\
\hline 1 & -0.150 & -0.100 & 0.150 & 0.100 & 0.280 \\
2 & & 0.250 & 0.100 & -0.250 & 0.170 \\
3 & & & 0.320 & -0.220 & -0.190 \\
4 & & & & -0.200 & 0.150 \\
5 & & & & & -0.180 \\
\hline \hline
\end{tabular}

Table 3: Estimated price parameters and standard deviations (in brackets)

\begin{tabular}{rrrrrr}
\hline \hline & 1 & \multicolumn{1}{c}{3} & \multicolumn{1}{c}{3} & \multicolumn{1}{c}{5} \\
\hline 1 & -0.1515 & -0.1006 & 0.1494 & 0.0997 & 0.2799 \\
& $(0.0140)$ & $(0.0106)$ & $(0.0103)$ & $(0.0090)$ & $(0.0087)$ \\
2 & & 0.2494 & 0.0999 & -0.2497 & 0.1699 \\
& & $(0.0176)$ & $(0.0129)$ & $(0.0102)$ & $(0.0102)$ \\
3 & & & 0.3200 & -0.2208 & -0.1891 \\
& & & $(0.0170)$ & $(0.0096)$ & $(0.0100)$ \\
4 & & & -0.1992 & 0.1490 \\
& & & & $(0.0117)$ & $(0.0079)$ \\
5 & & & & & -0.1803 \\
& & & & & \\
& & & & & \\
\hline \hline
\end{tabular}

\subsection{Bootstrap Inference}

The "wild bootstrap" draws new artificial responses based on the estimated model (1) with given sample $\left\{W_{i}, \tilde{X}_{i}, \tilde{P}_{i}\right\}_{i=1}^{N}$ and estimates $\hat{\alpha}^{j}, \hat{\beta}^{j}$ and $\hat{a}^{j k}, k, j=1, \ldots, d$. Denote an oversmoothed bandwidth $g$ with $g>h$ (obeying the needs of asymptotic theory), and let $h$ be the bandwidth giving us the desired smoothness in the original sample. The basic idea is now (cf. [7]) to use the estimated residuals from an estimate with bandwidth $g$,

$$
\hat{\varepsilon}_{i}^{j}=W_{i}^{j}-\frac{\hat{\alpha}^{j}\left(\tilde{X}_{i}\right)+\sum_{k=1}^{d} \hat{a}^{j k} \tilde{P}_{i}^{k}}{1-\sum_{k=1}^{d} \hat{\beta}^{k}\left(\tilde{X}_{i}\right) \tilde{P}_{i}^{k}} .
$$

to get wild bootstrap residuals $\varepsilon_{i}^{j *}$. Given them we create bootstrap samples $\left\{W_{i}^{*}, \tilde{X}_{i}, \tilde{P}_{i}\right\}_{i=1}^{N}$ by

$$
W_{i}^{j *}=\frac{\hat{\alpha}^{j}\left(\tilde{X}_{i}\right)+\sum_{k=1}^{d} \hat{a}^{j k} \tilde{P}_{i}^{k}}{1-\sum_{k=1}^{d} \hat{\beta}^{k}\left(\tilde{X}_{i}\right) \tilde{P}_{i}^{k}}+\varepsilon_{i}^{j *}
$$

for $i=1, \ldots, N$ and $j=1, \ldots, M-1$. Here, $\varepsilon_{i}^{j *}$ are bootstrap residuals that replicate desired properties of the distribution(s) of $\hat{\varepsilon}_{i}^{j}$. The $W_{i}^{M *}$ are generated via $\sum_{j=1}^{M} W_{i}^{j *}=1$. We can repeat this many times, estimate functions and parameter of interest for each bootstrap sample and use the bootstrap quantiles to construct 
point wise confidence bands for our estimates. Although it is well known that these bands can suffer from smoothing biases, and the proper choice of $g$ for a particular $h$ is problematic, they give a pretty good idea of the estimator's statistical behaviour.

There exist several strategies to obtain wild bootstrap residuals $\varepsilon_{i}^{j *}$. Typically, when no problem of censoring or other restrictions is faced, one may use

$$
\varepsilon_{i}^{j *}=u_{i} \cdot \hat{\varepsilon}_{i}^{j},
$$

with $u_{i}$ being a standard normal random scalar. Under the additional assumption of homoscedasticity, this can even be simplified to $\varepsilon_{i}^{j *}=u_{i} \cdot \hat{\sigma}_{\varepsilon}^{j}$, where $\sigma_{\varepsilon}^{j}$ is estimated from the residuals (23).

In our case, one may argue that such bootstrap errors may cause the bootstrap values of $W_{i}^{j *}$ to lie outside the admissible range of $[0,1]$ for budget shares. On the one hand, this may not matter much because the estimation algorithm does not control or force the constraint that $\hat{W}_{i}^{j} \in[0,1]$. However, given that some items are censored at zero expenditures, the bootstrap residuals may poorly reflect the true error distribution and misrepresent the confidence intervals, for example putting them outside $[0,1]$.

To address the possibility that the bootstrap inference is hampered by bootstrap budget-shares lying outside $[0,1]$, we introduce an alternative formulation of the wild bootstrap. Obviously, for the items with censored expenditures, we are faced with a conditionally asymmetric (to the right) error distribution. We thus consider an asymmetric distribution for $\varepsilon_{i}^{j *}$ given $\hat{\varepsilon}_{i}^{j}$ as follows. Generate bootstrap errors via

$$
\frac{\chi_{k}^{2}}{\sqrt{k}} \cdot \frac{\left|\hat{\varepsilon}_{i}^{j}\right|}{\sqrt{2}}-\frac{\left|\hat{\varepsilon}_{i}^{j}\right|}{\sqrt{2}} \cdot \sqrt{k} \leq\left|\hat{W}_{i}^{j}\right|,
$$

where $k \leq\left\lfloor\left(W_{i}^{j} / \hat{\varepsilon}_{i}^{j}\right)^{2} \cdot 2\right\rfloor$. In the case that $k$ is less than one, we draw the bootstrap residual $\varepsilon_{i}^{j *}$ from $\chi_{1}^{2} \cdot\left|\hat{W}_{i}^{j}\right|-\left|\hat{W}_{i}^{j}\right|$. Note that this fulfills $E\left[\varepsilon_{i}^{j *}\right]=0$ and $E\left[\left(\varepsilon_{i}^{j *}\right)^{2}\right]=E\left[\left(\hat{\varepsilon}_{i}^{j}\right)^{2}\right]$ for all $i$ and $j$. From (25), we have that, for positive $\hat{W}_{j}^{j}$, its bootstrap analog is always positive, too. This method leads automatically to confidence bands that hardly jut out of $[0,1]$ and are consequently narrower than those based on a simple normal bootstrap described above. In the empirical work below, we present confidence intervals based on this asymmetric wild bootstrap, and confidence intervals based on the regular wild bootstrap are available on request from the authors.

\subsection{Analysing Household Expenditures in Canada}

In our empirical study we use the same Canadian data as in [12] and [15] which come from public sources, see also [14]. The price and expenditure data are available for 12 years in 5 regions: Atlantic, Quebec, Ontario, Prairies and British Columbia. This yields 60 distinct price vectors, where prices are normalised in a way that all prices of the categories from Ontario in 1986 are one, i.e. $\tilde{p}_{O, 86}=(0, \ldots, 0)$, so these 189 observations define the base price vector and we use them to get the starting values. Note further, to achieve homogeneity we subtracted $p^{M}$, the price of the left-out expenditure category, from all other prices and total expenditure.

We use 6952 observations of rental-tenure unattached individuals aged between 25 and 64 with no dependants to minimise demographic variation in preferences. Our analysis includes annual total-expenditure in nine categories: food-in, food-out, rent, clothing, household operations, household furnishing and equipment, private transportation, public transportation and personal care. The left-out category is 
personal care, so that we get a system of eight expenditure share equations which depend on eight (normalised) log-prices and (normalised) log-total expenditure. These expenditure categories account for about three-quarters of the current consumption of the households in the sample. Summary statistics of the observations are given in Table 4.

Table 4: The Data

\begin{tabular}{llrrrr}
\hline \hline & & Min & Max & Mean & Std. \\
\hline expenditure shares & food-in & 0.00 & 0.63 & 0.17 & 0.09 \\
& food-out & 0.00 & 0.64 & 0.08 & 0.08 \\
& rent & 0.01 & 0.95 & 0.40 & 0.13 \\
& operations & 0.00 & 0.64 & 0.08 & 0.04 \\
& furnishing & 0.00 & 0.65 & 0.04 & 0.06 \\
& clothing & 0.00 & 0.53 & 0.09 & 0.06 \\
& private trans & 0.00 & 0.59 & 0.08 & 0.09 \\
& public trans & 0.00 & 0.34 & 0.04 & 0.04 \\
\hline $\log$-prices & food-in & -0.39 & 0.07 & -0.03 & 0.09 \\
& food-out & -0.42 & 0.25 & 0.05 & 0.12 \\
& rent & -0.35 & 0.14 & -0.12 & 0.15 \\
& operations & -0.28 & 0.10 & -0.04 & 0.08 \\
& furnishing & -0.16 & 0.21 & -0.03 & 0.09 \\
& clothing & -0.07 & 0.44 & 0.10 & 0.11 \\
& private trans & -0.51 & 0.30 & -0.09 & 0.18 \\
& public trans & -0.59 & 0.40 & 0.01 & 0.25 \\
\hline $\log$-total expenditure & & 3.03 & 6.26 & 4.61 & 0.45 \\
\hline \hline
\end{tabular}

We note that this choice of commodities is arbitrary: one could divide these goods into subcategories, or aggregate them up into larger categories. We choose these categories because they offer the finest gradation consistent with largest possible time span for the price data (finer gradations of price data are available, but for shorter periods of time). Another advantage of this choice of commodities is that they are directly comparable with [15].

As noted above, when $\tilde{p}=(0, \ldots, 0)$ (as it does for observations in Ontario 1986), the price effects in expenditure shares amount to zero, yielding

$$
\mathbf{w}(\mathbf{p}, x)=\mathbf{w}(\tilde{\mathbf{p}}, \tilde{x})=\mathbf{f}(\tilde{x})=\mathbf{f}(x),
$$

which we will refer to as the vector of Engel curves. The estimated Engel curves of all expenditure categories can be found in Figure 2 and 3 as solid lines, where the horizontal axes refer to $\tilde{x}$, i.e. the $\log$ total expenditures minus $p_{M}$, the $\log$-prize of item $M$.

We include pointwise $90 \%$ confidence intervals which we calculated as described in Section 4.2 with heteroscedastic error terms and 500 bootstrap iterations using our new conditionally asymmetric wild bootstrap procedure. To generate the bootstrap samples we used the bandwidth $g=1.2 h$ (giving rather smooth confidence bands) and in the estimation of the functions $\alpha^{j}$ the bandwidth $h=0.1725$. The nonparametric part is estimated over a grid of 30 equispaced points using the Gaussian kernel, and it converged in our setting after about 15 iterations. In all figures, the estimates are compared to the parametric estimators of [1] and the semiparametric estimators (assuming a partial linear cost function with Slutsky symmetry) of [15]. 
Table 5: Estimated symmetric price effects $a^{j k}$ (with bootstrap generated standard deviations in brackets)

\begin{tabular}{|c|c|c|c|c|c|c|c|}
\hline food-in & food-out & rent & oper & furn & clothing & priv tr & pub tr \\
\hline $\begin{array}{rr}\text { food-in }-0.026 \\
(0.036)\end{array}$ & $\begin{array}{c}0.013 \\
(0.018)\end{array}$ & $\begin{array}{r}-0.006 \\
(0.012)\end{array}$ & $\begin{array}{r}-0.008 \\
(0.019)\end{array}$ & $\begin{array}{c}0.009 \\
(0.014)\end{array}$ & $\begin{array}{l}0.006 \\
(0.015)\end{array}$ & $\begin{array}{c}0.037 \\
(0.007)\end{array}$ & $\begin{array}{r}-0.058 \\
(0.006)\end{array}$ \\
\hline food-out & $\begin{array}{r}-0.035 \\
(0.014)\end{array}$ & $\begin{array}{r}0.047 \\
(0.007)\end{array}$ & $\begin{array}{c}0.012 \\
(0.012)\end{array}$ & $\begin{array}{r}-0.002 \\
(0.010)\end{array}$ & $\begin{array}{r}-0.069 \\
(0.009)\end{array}$ & $\begin{array}{r}0.001 \\
(0.005)\end{array}$ & $\begin{array}{r}-0.045 \\
(0.005)\end{array}$ \\
\hline rent & & $\begin{array}{r}0.186 \\
(0.017)\end{array}$ & $\begin{array}{c}0.023 \\
(0.007)\end{array}$ & $\begin{array}{r}-0.026 \\
(0.005)\end{array}$ & $\begin{array}{r}-0.021 \\
(0.008)\end{array}$ & $\begin{array}{r}-0.036 \\
(0.006)\end{array}$ & $\begin{array}{r}0.087 \\
(0.005)\end{array}$ \\
\hline oper & & & $\begin{array}{l}0.040 \\
(0.017)\end{array}$ & $\begin{array}{r}0.010 \\
(0.011)\end{array}$ & $\begin{array}{r}-0.016 \\
(0.011)\end{array}$ & $\begin{array}{r}-0.029 \\
(0.004)\end{array}$ & $\begin{array}{r}0.023 \\
(0.005)\end{array}$ \\
\hline furn & & & & $\begin{array}{r}-0.038 \\
(0.016)\end{array}$ & $\begin{array}{r}0.026 \\
(0.009)\end{array}$ & $\begin{array}{r}-0.017 \\
(0.004)\end{array}$ & $\begin{array}{r}-0.024 \\
(0.004)\end{array}$ \\
\hline clothing & & & & & $\begin{array}{r}0.005 \\
(0.010)\end{array}$ & $\begin{array}{r}-0.002 \\
(0.005)\end{array}$ & $\begin{array}{r}-0.014 \\
(0.004)\end{array}$ \\
\hline priv tr & & & & & & $\begin{array}{r}0.002 \\
(0.006)\end{array}$ & $\begin{array}{r}0.006 \\
(0.003)\end{array}$ \\
\hline pub tr & & & & & & & $\begin{array}{r}-0.011 \\
(0.003)\end{array}$ \\
\hline
\end{tabular}

The estimated Engel curves in the figures hold no surprises relative to other semiparametric work. In terms of local regularity, the estimated values of budget-shares lie entirely within $[0,1]$. Although we do not assess the global regularity of our estimates or estimator, it is comforting that estimated budget shares satisfy this condition locally.

Food-at-home and food-out are strong necessities and luxuries, respectively, with nearly linear Engel curves in both cases. The near-linearity of these Engel curves has been observed in a large number of empirical investigations, including [1]. Some curvature is observed in the rent and clothing equations, especially near the bottom of the distribution. This curvature is noted in semiparametric work, such as [15] and [12]. The most curvature is noted in smaller budget shares like household operation, private transportation and public transportation. Although the curvature in the latter equation does not seem very statistically significant given the confidence intervals (shown with black dots), the curvature in household operation seems quite strong, and that in private transportation seems decidedly non-quadratic.

Thus, the estimated Engel curves are plausible and have some evidence of complexity beyond the quadratic form of [1]. In comparison with [15], the present approach has an important computational advantage: it is based entirely on observed regressors, and so does not require any numerical inversions to generate a latent regressor. In comparison with [12], the present approach has an important interpretational difference: whereas [12] must interpret model error terms as unobserved preference heterogeneneity parameters, the present approach is based on the more standard view of error terms as measurement or other non-behavioural error.

Table 5 gives the estimated symmetric price parameters and in brackets the bootstrapped standard deviations. These estimated price effects are in the plausible range, and are similar to those found in [15].

The varying-coefficients extension is similarly easy to implement. We use the same bandwidths (which are driven in the main by the fit for $\mathbf{f}(\tilde{x})$ ) as in the fixedcoefficients case. The estimated Engel curves are almost identical to those found in the fixed-coefficients case, with some small deviations in the tails. For the sake 

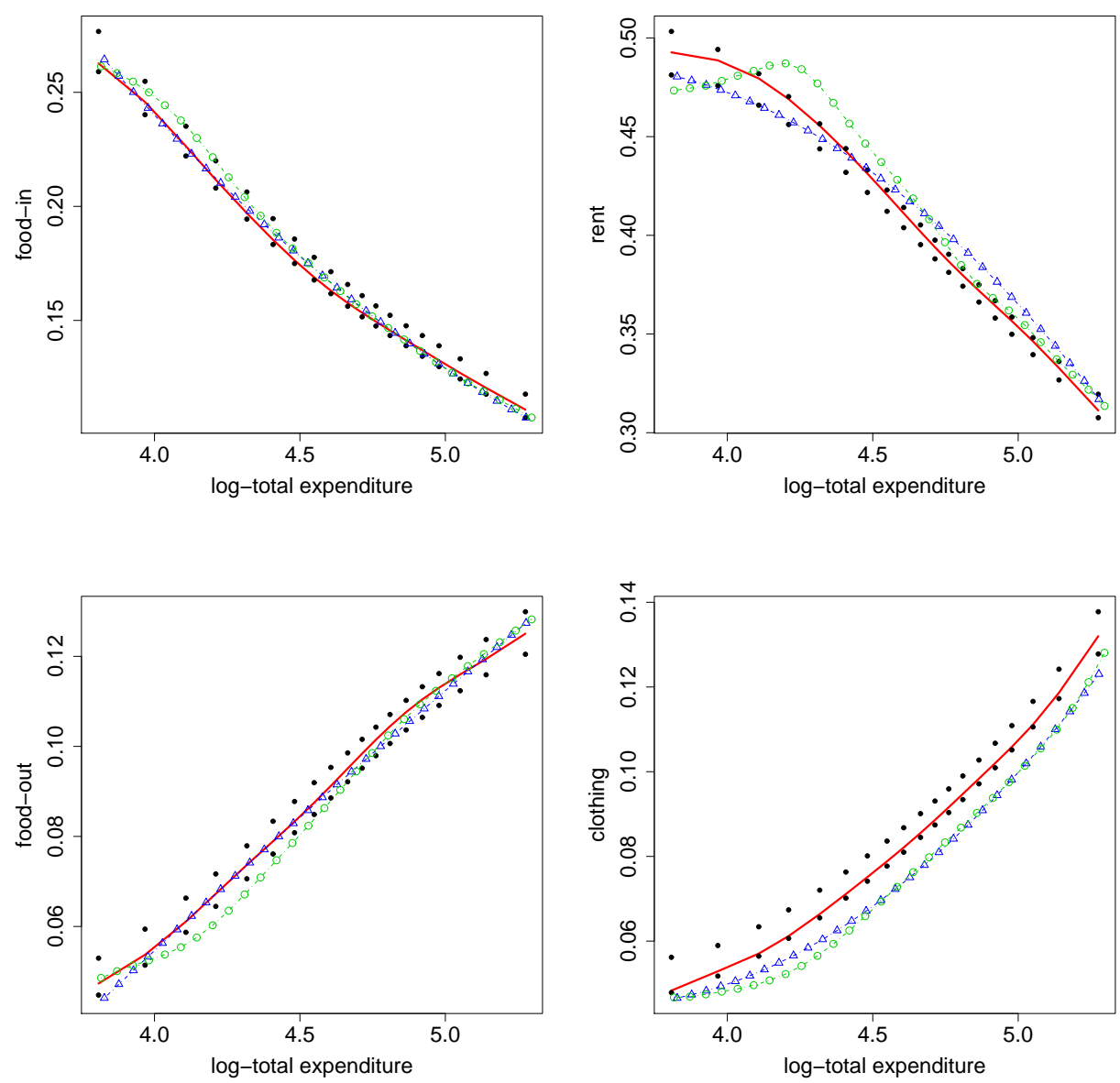

Figure 2: Estimates of food-in, food-out, rent, and clothing (solid line) with 90\% pointwise confidence bands, together with estimates using [1] (blue with triangles) and [15] (green with circles). 

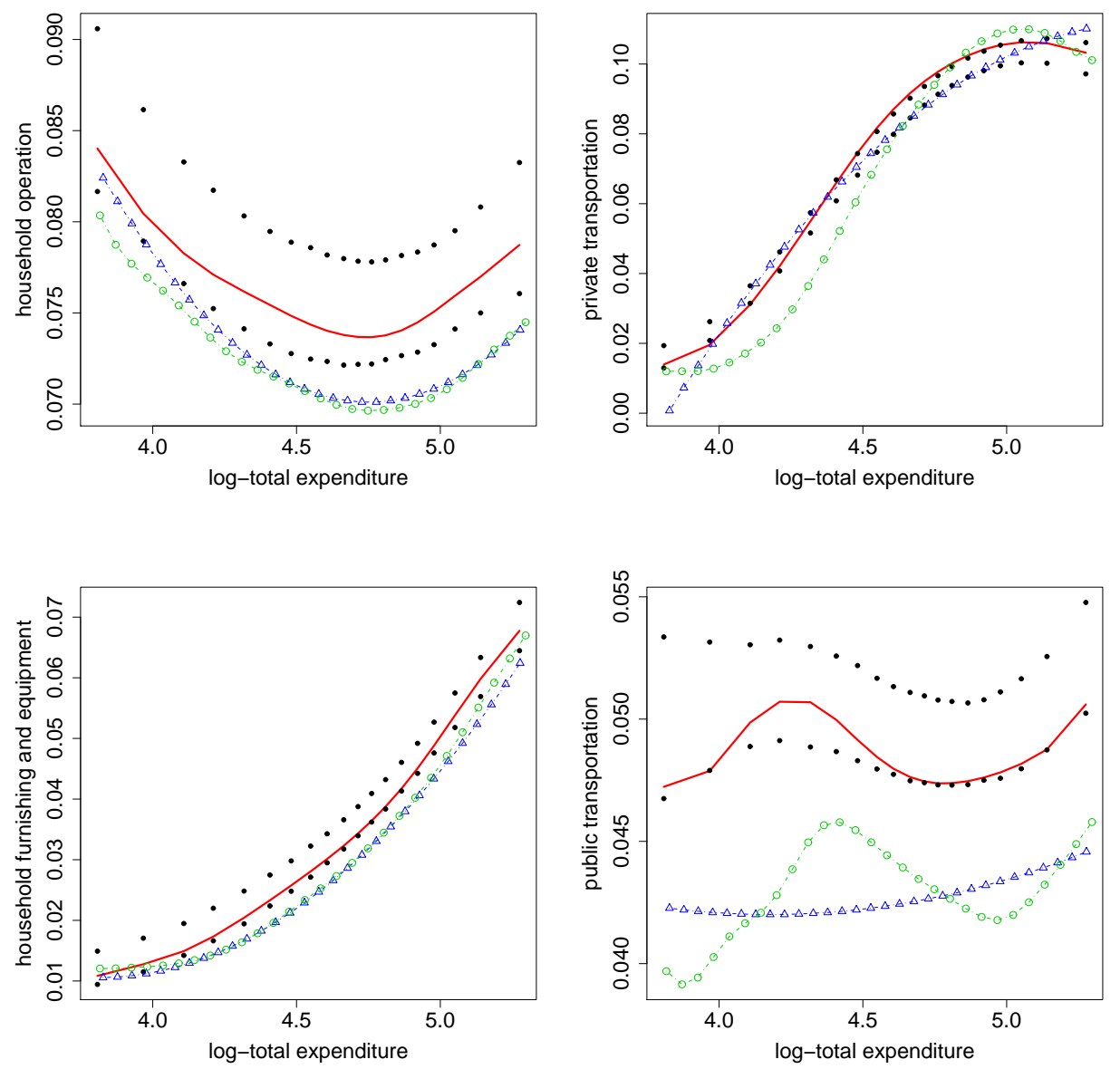

Figure 3: Estimates of household operations, furnishing and equipment, private and public transportation (solid line) with $90 \%$ pointwise confidence bands, together with estimates using [1] (blue with triangles) and [15] (green with circles). 
of brevity, we do not present those results here, but they are available on request from the authors. Depending on the bandwidth, the estimated price parameters evaluated at median log-expenditure are statistically indistinguishable from those of the fixed-coefficients model, but their estimated variance is much greater. In particular, we see approximately twice the standard errors for estimated parameters evaluated at median expenditures relative to their fixed-coefficients counterparts.

\section{Conclusions}

We propose a model which starts with the indirect utility function and implies a consumer demand system that has parametric log-price effects and nonparametric log-total expenditure effects. Furthermore, we avoid the curse of dimensionality typically associated in fully nonparametric estimation of consumer demand since the nonparametric part of the model is only one dimensional in log-total expenditure. The model is easily restricted to satisfy the rationality conditions of homogeneity and Slutsky symmetry.

A detailed explanation of the estimation procedure shows the working of the method, and a simulation shows finite sample performance. We provide a new wild bootstrap procedure that allows for conditional asymmetries and guarantees positive shares. We then show the finite sample performance of our estimators in a simulation study, and finally apply our method to Canadian expenditure data. Extensions to tackle with possibly endogenous expenditures are possible via the approach proposed in [21].

The application of this model to Canadian price and expenditure data shows not only the potential of the model but also suggests that some expenditure shares more complex than the linear ones in the popular AID [4] and Translog [10] demand models. The simulation study reveals further that it is also possible to estimate functions which are difficult to estimate [9], such as those with flat plateaus in the intermediate range or with bumps.

\section{Appendix: Restricted Least Squares for a Symmetric Matrix A}

Recall that to estimate the symmetric parameters $a^{j k}, j, k=1, \ldots, d$, we use equation (6) and get, with some algebra, for a single individual $i$

$$
W_{i}^{j} \cdot\left(1-\sum_{k=1}^{d} \beta_{i}^{k} \tilde{P}_{i}^{k}\right)-\alpha_{i}^{j}=\sum_{k=1}^{d} a^{j k} \tilde{P}_{i}^{k} .
$$

Here, the parameters $\alpha_{i}^{j}=\alpha^{j}\left(\tilde{X}_{i}\right)$ are related to the functions $f^{j}$ at the point $\tilde{X}_{i}$ and the parameters $\beta_{i}^{j}=\beta^{j}\left(\tilde{X}_{i}\right)$ to its first derivatives. Defining $(\boldsymbol{W})_{i j}:=$ $W_{i}^{j} \cdot\left(1-\sum_{k=1}^{d} \beta_{i}^{k} \tilde{P}_{i}^{k}\right)-\alpha_{i}^{j},(\boldsymbol{P})_{i k}:=\tilde{P}_{i}^{k}$ and $(\boldsymbol{A})_{k j}:=a^{k j}$ we can formulate equation (26) using matrix notation:

$$
\boldsymbol{W}=\boldsymbol{P} \cdot \boldsymbol{A},
$$

where $\boldsymbol{W}, \boldsymbol{P}$ are $N \times d$ matrices and $\boldsymbol{A}$ a $d \times d$ symmetric matrix. Note that it is not necessary to start in the model description (1) with symmetric parameters $a^{j k}$. However, when we start with arbitrary parameters we will end nevertheless in (27) with a symmetric parameter matrix. More specific, we get for (26):

$$
W_{i}^{j} \cdot\left(1-\sum_{k=1}^{d} \beta_{i}^{k} \tilde{P}_{i}^{k}\right)-\alpha_{i}^{j}=1 / 2 \sum_{k=1}^{d}\left(a^{j k}+a^{k j}\right) \tilde{P}_{i}^{k}
$$


and in matrix notation

$$
\boldsymbol{W}=\frac{1}{2} \boldsymbol{P}\left(\boldsymbol{A}+\boldsymbol{A}^{t}\right)
$$

Obviously, $\boldsymbol{A}+\boldsymbol{A}^{t}$ is symmetric and we would have an identification problem for nonsymmetric $\boldsymbol{A}$. In other words, even if one does not require symmetry from the beginning, only symmetry of $\boldsymbol{A}$ makes the estimation problem identifiable.

Next, to calculate the unknown matrix $\boldsymbol{A}$ in equation (27) we should not use the standard least square method but want directly make use of the symmetry of $\boldsymbol{A}$. Denote $w^{i j}=(\boldsymbol{W})_{i j}$ and $w^{j}$ the $\mathrm{j}$-th column of $\boldsymbol{W}, p^{i j}=(\boldsymbol{P})_{i j}, p_{i}$ the $\mathrm{i}$-th row and $p^{j}$ the $\mathrm{j}$-th column of the price-matrix $\boldsymbol{P}$ and $a^{j}$ also the $\mathrm{j}$-th row of $\boldsymbol{A}$. Note that the symmetric matrix $\boldsymbol{A}$ obtains only $\left(d^{2}+d\right) / 2$ different parameters which are found for example in the lower triangular part, including the diagonal elements

$$
\boldsymbol{A}=\left(\begin{array}{cccc}
a_{1} & a_{2} & \ldots & a_{d} \\
a_{2} & a_{d+1} & \ldots & a_{2 d-1} \\
\ldots & \ldots & \ldots & \ldots \\
a_{d} & a_{2 d-1} & \ldots & a_{\left(d^{2}+d\right) / 2}
\end{array}\right)=\left(\begin{array}{cccc}
a^{11} & a^{12} & \ldots & a^{1 d} \\
a^{21} & a^{22} & \ldots & a^{2 d} \\
\ldots & \ldots & \ldots & \ldots \\
a^{d 1} & a^{d 2} & \ldots & a^{d d}
\end{array}\right)
$$

Let $\boldsymbol{a}_{p}$ be the one-dimensional array formed by these parameters,

$$
\boldsymbol{a}_{p}=\left(a_{1}, \ldots, a_{\left(d^{2}+d\right) / 2}\right),
$$

then we have to find the vector $\boldsymbol{a}_{p}$ that minimises

$$
S:=\sum_{j=1}^{d} \sum_{i=1}^{N}\left(w_{i j}-\left\langle p_{i}, a^{j}\right\rangle\right)^{2} \longrightarrow \min _{\boldsymbol{a}_{p}} .
$$

We obtain by differentiation of (28) with respect to all elements of $\boldsymbol{a}_{p}$ the linear equation system $\boldsymbol{B} \boldsymbol{a}_{p}=\boldsymbol{c}$ which can be solved by standard methods. In detail, we construct the coefficient matrix $\boldsymbol{B}$ and the constant vector $\boldsymbol{c}$ in the following way. For the diagonal elements of $\boldsymbol{A}$ we get

$$
\frac{\partial S}{\partial a^{l l}}=-2 \sum_{i=1}^{N}\left(w^{i l}-\left\langle p_{i}, a^{l}\right\rangle\right) p^{i l} \stackrel{!}{=} 0
$$

for $l=1, \ldots, d$. This is equivalent to

$$
\begin{aligned}
\sum_{i=1}^{N} w^{i l} p^{i l} & =\sum_{i=1}^{N}\left\langle p_{i}, a^{l}\right\rangle p^{i l} \\
& =\sum_{i=1}^{N} \sum_{j=1}^{d} p^{i j} a^{j l} p^{i l}=\sum_{j=1}^{d} \sum_{i=1}^{N} p^{i j} p^{i l} a^{j l}
\end{aligned}
$$

what gives

$$
\left\langle w^{l}, p^{l}\right\rangle=\sum_{j=1}^{d}\left\langle p^{j}, p^{l}\right\rangle a^{j l} .
$$

For the off-diagonal elements we obtain

$$
\frac{\partial S}{\partial a^{k l}}=-2 \sum_{i=1}^{N}\left(w^{i l}-\left\langle p_{i}, a^{l}\right\rangle\right) p^{i k}-2 \sum_{i=1}^{N}\left(w^{i k}-\left\langle p_{i}, a^{k}\right\rangle\right) p^{i l} \stackrel{!}{=} 0
$$

for $k, l=1, \ldots, d$ and $k>l$. This is equivalent to

$$
\left\langle w^{l}, p^{k}\right\rangle+\left\langle w^{k}, p^{l}\right\rangle=\sum_{j=1}^{d}\left\langle p^{j}, p^{k}\right\rangle a^{j l}+\sum_{j=1}^{d}\left\langle p^{j}, p^{l}\right\rangle a^{j k} .
$$


The t-th entry in $\boldsymbol{c}$ we get now by the left hand side of (29), when $\boldsymbol{a}_{p}(t)$ corresponds to a diagonal element of $\boldsymbol{A}$, or by the left hand side of (30) otherwise. Note that $t=k+(2 d-l)(l-1) / 2$ for $k \geq l$ and $t=1, \ldots,\left(d^{2}+d\right) / 2$. The $\mathrm{t}-\mathrm{th}$ row of $\boldsymbol{B}$ we obtain by the right hand side of (29) or (30), where obviously the factors of the $a^{j k}$ are our searched coefficients. So an explicit solution is available such that no iteration is necessary for estimating $\mathbf{A}$ in the partial linear model case. This is exactly the reason for both, the much smaller variance of the resulting estimates in practice and the much higher speed of the algorithm for estimating model (1) compared to the one for estimating the varying coefficients model (3).

Example For the simple case $d=3$ we get the linear equation system $\boldsymbol{B} \boldsymbol{a}_{p}=\boldsymbol{c}$ with $\boldsymbol{a}_{p}=\left(a^{11}, a^{21}, a^{31}, a^{22}, a^{32}, a^{33}\right)$, the coefficient matrix $\boldsymbol{B}$

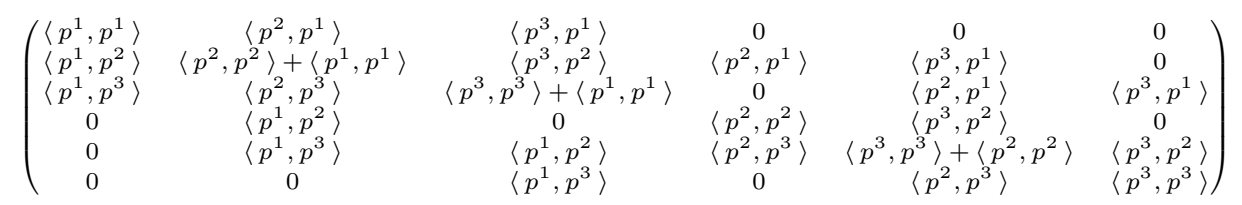

and vector $c$ as

$$
\left(\begin{array}{c}
\left\langle w^{1}, p^{1}\right\rangle \\
\left\langle w^{1}, p^{2}\right\rangle+\left\langle w^{2}, p^{1}\right\rangle \\
\left\langle w^{1}, p^{3}\right\rangle+\left\langle w^{3}, p^{1}\right\rangle \\
\left\langle w^{2}, p^{2}\right\rangle \\
\left\langle w^{2}, p^{3}\right\rangle+\left\langle w^{3}, p^{2}\right\rangle \\
\left\langle w^{3}, p^{3}\right\rangle
\end{array}\right)
$$

\section{References}

[1] Banks, J., Blundell, R., and Lewbel, A., 1997. Quadratic Engel curves and consumer demand. Review of Economics and Statistics, 79 527-539.

[2] Blundell, R., Chen, X., and Kristensen, D., 2003. Semi-nonparametric IV estimation of shape-invariant Engel curves. (Centre for Microdata Methods and Practice, working paper CWP15/03).

[3] Blundell, R., Duncan, A., and Pendakur, K., 1998. Semiparametric estimation of consumer demand. Journal of Applied Econometrics, 13 435-461.

[4] Deaton, A. and Muellbauer, J., 1980. An almost ideal demand system. American Economic Review, 70 312-326.

[5] Fan, J. and Zhang, W., 1999. Statistical estimation in varying coefficient models. Annals of Statistics, 27 1491-1518.

[6] Haag, B.R., Hoderlein, S., and Pendakur, K., 2006. Testing and imposing Slutsky symmetry in nonparametric demand systems. (Simon Fraser University, working paper).

[7] Härdle, W. and Marron, J.S., 1991. Bootstrap simultaneous error bars for nonparametric regression. The Annals of Statistics, 19 778-796.

[8] Härdle, W., Müller, M., Sperlich, S., and Werwatz A., 2004. Nonparametric and semiparametric models. Springer Verlag, Berlin.

[9] Hastie, T.J. and Tibshirani, R.J., 1984. Generalized aditive models. (Department of Statistics, Stanford University, Technical Report 98). 
[10] Jorgensen, D., Lau, L., and Stoker, T., 1980. Welfare comparison and exact aggregation. American Economic Review, 70 268-272.

[11] Lewbel, A., 2000. Consumer demand systems and household equivalence scales, Handbook of Applied Econometrics, Volume II: Microeconomics. Blackwell Publishers Ltd, Oxford.

[12] Lewbel, A. and Pendakur, K., 2009. Tricks with Hicks: The EASI demand system, American Economic Review. (forthcoming).

[13] Moral-Arce, I., Rodríguez-Póo, J.M. and Sperlich, S., 2007. Feasible semiparametric estimation of censored expenditure equations, Preprint, University of Göttingen, ZfS-2007-13.

[14] Pendakur, K., 2002. Taking prices seriously in the measurement of inequality. Journal of Public Economics, 86 47-69.

[15] Pendakur, K. and Sperlich, S., 2009. Semiparametric estimation of consumer demand systems in real expenditure. Journal of Applied Econometrics. (forthcoming).

[16] Politis, D.N., Romano, J.P., and Wolf, M., 1999. Subsampling. Springer, New York.

[17] Pollack, R. and Wales, T., 1991. Demand system specification and estimation. Oxford University Press, New York.

[18] Rodríguez-Póo, J.M., Sperlich, S. and Vieu, P., 2003. Semiparametric estimation of weak and strong separable sodels. Econometric Theory, 19 1008-1039.

[19] Sarmiento, C., 2005. A varying coefficient approach to global flexibility in demand analysis: a semiparametric approximation. American Journal of Agricultural Economics, 87 38-47.

[20] Severini, T.A. and Wong, W.W., 1992. Profile likelihood and conditionally parametric models. Annals of Statistics, 4 1768-1802.

[21] Sperlich, S., 2009. A note on nonparametric estimation with predicted variables. The Econometrics Journal. (forthcoming).

[22] Varian, H.R., 1978. Microeconomic Analysis. Norton, New York. 